\title{
Correction to: Impact of Data Augmentation on Retinal OCT Image Segmentation for Diabetic Macular Edema Analysis
}

Daniel Bar-David, Laura Bar-David, Shiri Soudry, and Anath Fischer

\begin{abstract}
Correction to:
Chapter "Impact of Data Augmentation on Retinal OCT Image Segmentation for Diabetic Macular Edema Analysis" in: H. Fu et al. (Eds.): Ophthalmic Medical Image Analysis, LNCS 12970, https://doi.org/10.1007/978-3-030-87000-3_16
\end{abstract}

The original version of this chapter was revised. The last name of an author was incorrect. The author's last name has been corrected to "Soudry". 